\title{
Francisella philomiragia adenitis and pulmonary nodules in a child with chronic granulomatous disease
}

\author{
Timothy L Mailman MD, Matthias H Schmidt MD
}

\begin{abstract}
TL Mailman, MH Schmidt. Francisella philomiragia adenitis and pulmonary nodules in a child with chronic granulomatous disease. Can J Infect Dis Med Microbiol 2005;16(4):245-248.

Francisella philomiragia is a rare and opportunistic pathogen capable of producing invasive infection in patients with compromised neutrophil function and in patients that have survived a near-drowning. A case of F philomiragia adenitis and lung nodules, refractory to cephalosporin therapy, is reported in a 10 -year-old boy with chronic granulomatous disease following a facial abrasion from a saltwater crab. To the authors' knowledge, this is the first Canadian clinical isolate to be reported. Genus and species identification was confirmed via $16 \mathrm{~S}$ ribosomal RNA sequence analysis. A literature review revealed three groups at risk of $F$ philomiragia infection: young patients with chronic granulomatous disease; adults with hematogenous malignancy; and near-drowning patients. Pneumonia, fever without an apparent source and sepsis are the main clinical presentations. Invasive procedures may be required to isolate this organism and ensure appropriate antimicrobial therapy. Limited awareness of F philomiragia has led to delayed identification, patient death and misidentification as Francisella tularensis - a biosafety level three pathogen and potential bioterrorism agent.
\end{abstract}

Key Words: Chronic granulomatous disease; Francisella philomiragia

The present article describes the first case of Francisella 1 philomiragia infection reported in Canada, which manifested as adenitis and pulmonary infection in a patient with chronic granulomatous disease (CGD). As a member of the Francisella genus, F philomiragia is a strictly aerobic, Gram-negative coccobacillus. Unlike its more virulent relative Francisella tularensis, however, F philomiragia is an opportunist, causing pneumonia and systemic infection almost exclusively in immunocompromised patients or following near-drowning. Limited awareness of this organism has led to delayed diagnosis, therapeutic failures and poor outcomes. Confusion with $F$ tularensis may lead to false biohazard alarms, as occurred in the present case.

\section{CASE PRESENTATION}

A 10-year-old boy with CGD and on long-term itraconazole and trimethoprim-sulfamethoxazole (TMP-SMX) prophylaxis presented with a two-week history of fatigue and intermittent fever, followed by the appearance of tender, left-sided submandibular swelling. He subsequently developed a nonproductive cough and was brought to his physician for assessment. His medical history noted that he had never travelled outside of

\section{Adénite à Francisella philomiragia et nodules pulmonaires chez un enfant atteint de granulo- matose familiale chronique}

\begin{abstract}
Francisella philomiragia est un agent pathogène rare et opportuniste, capable de causer une infection invasive chez les patients qui présentent un affaiblissement des neutrophiles et chez ceux qui ont survécu à une quasi-noyade. Voici un cas d'adénite à $F$. philomiragia et de nodules pulmonaires réfractaires à la céphalosporine chez un garçonnet de 10 ans, atteint de granulomatose familiale chronique, qui a subi une lésion du visage par un crabe de mer. D'après les auteurs, il s'agirait là du premier isolat clinique signalé au Canada. L'identification du genre et de l'espèce de microbe a été confirmée par l'analyse de la séquence de l'ARN ribosomique $16 \mathrm{~S}$. Selon la documentation, trois groupes de personnes sont sensibles aux infections à $F$. philomiragia : les jeunes atteints de granulomatose familiale chronique, les adultes atteints de tumeurs malignes hématogènes et les personnes ayant survécu à une quasi-noyade. Pneumonie, fièvre sans source apparente d'infection et septicémie : voilà les principales manifestations cliniques. Il peut être nécessaire de recourir à des examens effractifs pour isoler le micro-organisme et déterminer un traitement antimicrobien approprié. Le manque de sensibilisation à F. philomiragia a conduit à un retard d'identification du microbe en cause, à la mort du patient et à l'identification erronée de Francisella tularensis, pathogène de troisième degré en matière de biosécurité et agent potentiel de menace bioterroriste.
\end{abstract}

Atlantic Canada and had no animal contacts. Just before the illness, a friend had tossed a saltwater crab at the patient, striking him on the left cheek and leaving an abrasion.

Initial physical examination noted a febrile, tired-appearing boy with a dry cough and no respiratory distress. Several oral ulcerations were noted, and palpation of the submandibular swelling revealed a mildly tender $2 \mathrm{~cm}$ left-sided submandibular lymph node. Crackles were detected at the right lung base. Examination was otherwise normal. A chest $\mathrm{x}$-ray was interpreted as normal. On complete blood count testing, the peripheral leukocyte count was $15 \times 10^{9} / \mathrm{L}$ with $77 \%$ neutrophils. Blood and urine cultures were collected.

Hospital admission was arranged, and empirical therapy with intravenous cefuroxime and oral azithromycin was initiated. Fever persisted over the next $72 \mathrm{~h}$, prompting the replacement of cefuroxime with intravenous cefotaxime. Blood and urine cultures were reported as negative. Despite broad-spectrum antimicrobial therapy, the patient failed to defervesce, had ongoing tenderness of the node and, of more concern, became tachypneic. Examination of a follow-up chest $\mathrm{x}$-ray revealed a small effusion at the right lung base.

IWK Health Centre, Dalhousie University, Halifax, Nova Scotia

Correspondence: Dr Timothy L Mailman, Department of Pediatrics, IWK Health Centre, Dalhousie University,

5850 University Avenue, Halifax, Nova Scotia B3K 6R8. Telephone 902-470-7892, fax 902-470-7046, e-mail tmailman@dal.ca

Received for publication July 28, 2004. Accepted January 17, 2005 


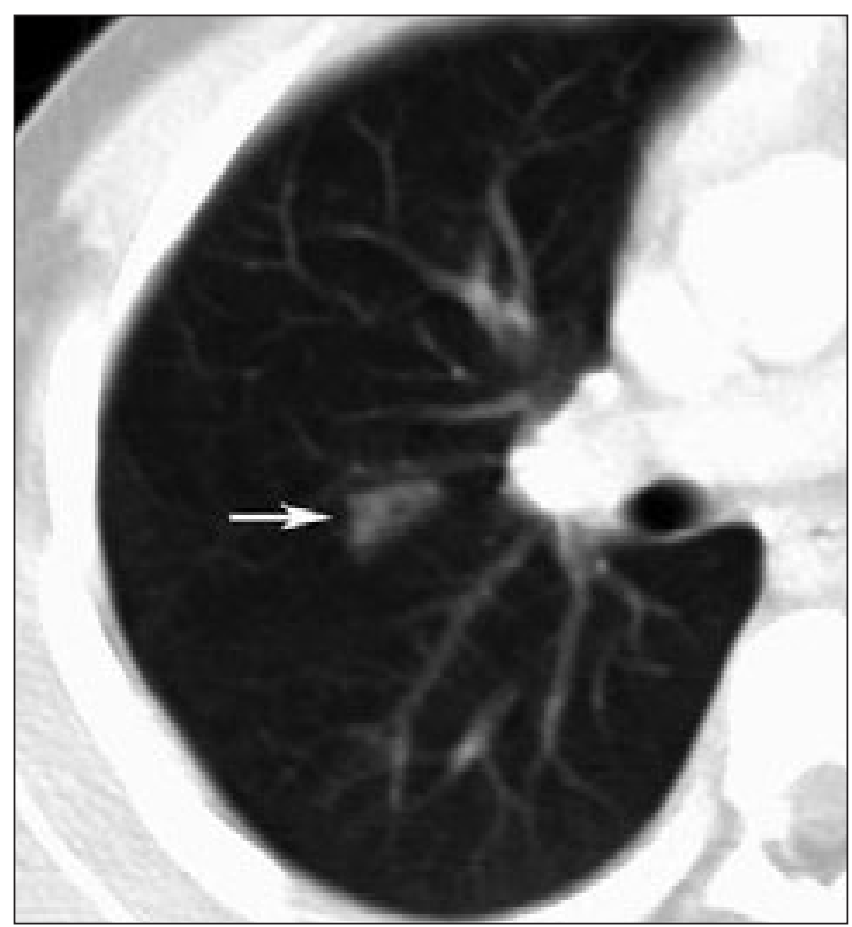

Figure 1) Computed tomography examination of the thorax with intravenous contrast enhancement shows a small triangular airspace opacity in the posterior segment of the right upper lobe (arrow)

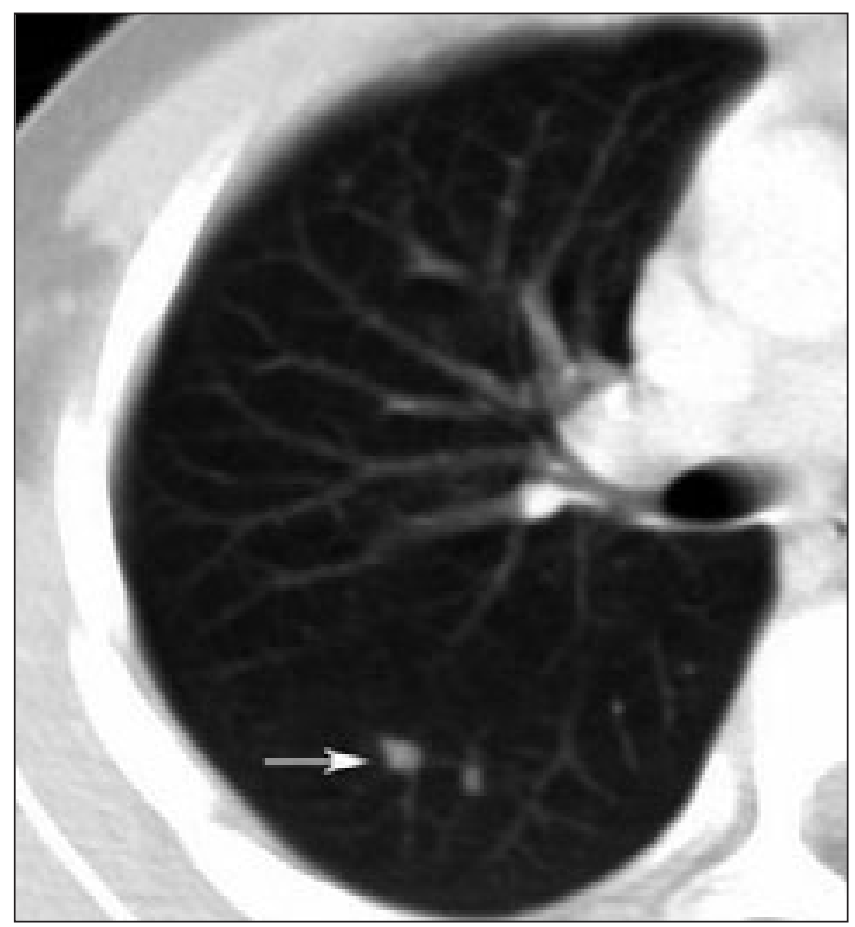

Figure 2) Computed tomography image of the right lung demonstrating pulmonary nodules (arrow)

A computed tomography scan of the neck and thorax was performed. An inspection of the images noted extensive noncaseating enlargement of several submandibular and deep axillary nodes and a wedge-shaped consolidation in the right lung (Figure 1) accompanied by several pulmonary nodules (Figure 2).

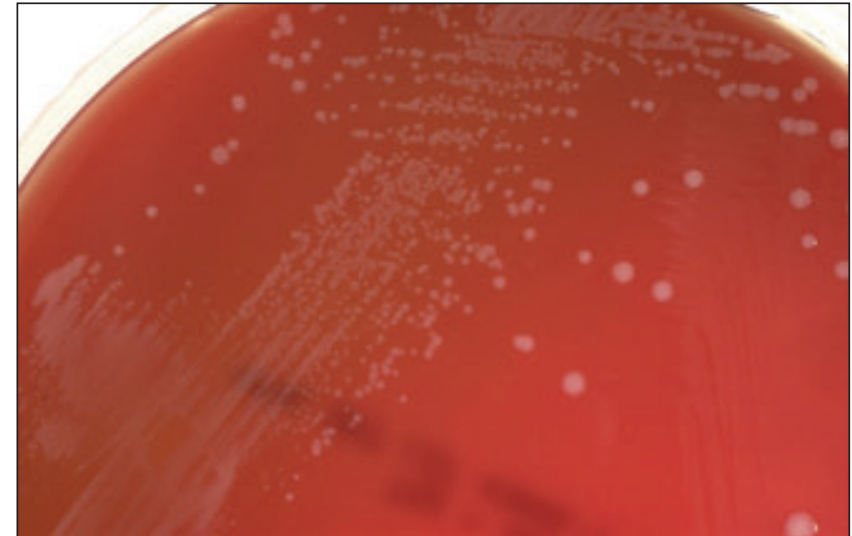

Figure 3) Typical Francisella philomiragia colonies on sheep blood agar have a smooth, white and convex appearance

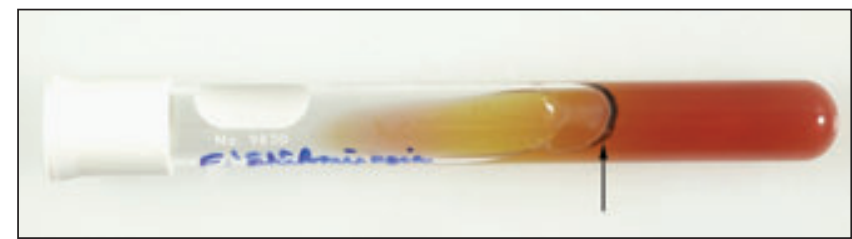

Figure 4) This thin line of hydrogen sulfide production (arrow) in a triple sugar iron slant is one of the key features in the identification of Francisella philomiragia

A submandibular node was surgically excised, sent for histological examination and cultured for bacteria, mycobacteria and viruses. The histopathology report described a mildly reactive histiocytosis of the node without granuloma formation. Acid-fast and Gram stains of tissue were negative. Within $48 \mathrm{~h}$, tiny, weakly catalase-positive colonies were observed on sheep blood agar incubated aerobically and chocolate agar incubated in $5 \% \mathrm{CO}_{2}$ (Figure 3 ).

Microscopy of Gram-stained organisms noted bizarre, amorphous, Gram-negative material. Closer examination revealed very tiny, poorly stained, Gram-negative coccobacilli. These were initially (and incorrectly) interpreted as oxidase-negative, prompting consideration of the organism as $F$ tularensis, which is a biohazard level three agent. The organism was transferred to a level three laboratory for definitive identification, and the patient's antibiotic regimen was changed to ciprofloxacin monotherapy.

Four laboratory staff had handled the organism on an open bench. Due to potential F tularensis exposure, these staff members were assessed at the infectious diseases clinic of the Queen Elizabeth II Health Sciences Centre (Nova Scotia) and placed on prophylactic doxycycline pending identification of the organism.

Subsequently, the oxidase test was repeated and determined to be weakly positive (ruling out $F$ tularensis). The organism grew as a nonmotile strict aerobe that produced hydrogen sulfide on triple sugar iron agar slants (Figure 4). Acid production from glucose and sucrose was demonstrated and gelatin was slowly hydrolyzed. The isolate was tentatively identified as $F$ philomiragia and confirmed as such by $16 \mathrm{~S}$ ribosomal RNA sequencing.

While susceptibility testing remains nonstandardized for Francisella species, both Kirby-Bauer and broth microdilution 
TABLE 1

Demographic and clinical features of patients infected with Francisella philomiragia

\begin{tabular}{|c|c|c|c|c|c|c|c|}
\hline Patient & $\begin{array}{c}\text { Age/sex } \\
\text { (reference) }\end{array}$ & $\begin{array}{c}\text { Date of } \\
\text { isolation }\end{array}$ & $\begin{array}{l}\text { Geographic } \\
\text { source }\end{array}$ & $\begin{array}{l}\text { Clinical } \\
\text { source }\end{array}$ & $\begin{array}{l}\text { Underlying } \\
\text { illness }\end{array}$ & $\begin{array}{c}\text { Clinical } \\
\text { presentation }\end{array}$ & Treatment \\
\hline 1 & $18 /$ male (1) & $12 / 27 / 74$ & California, USA & Lung biopsy & CGD & Pneumonia & Chloramphenicol, sulfisoxazole, penicillin G \\
\hline 2 & $39 /$ male $(1)$ & $10 / 21 / 76$ & Colorado, USA & Pleural fluid & Pleural effusion & Fever & None \\
\hline 3 & $\mathrm{NA} /$ male (1) & $10 / 04 / 77$ & New York, USA & Blood & Near-drowning & NA & NA \\
\hline 4 & $39 /$ male $(1)$ & $9 / 13 / 78$ & California, USA & Blood & Near-drowning & Pneumonia & Cephalothin, gentamicin \\
\hline 5 & 6/male (1) & July 1979 & Switzerland & $\begin{array}{l}\text { Blood, bone } \\
\text { marrow, } \\
\text { ascitic fluid }\end{array}$ & CGD & Fever, sepsis & $\begin{array}{l}\text { Cefotaxime, amikacin, trimethoprim- } \\
\text { sulfamethoxazole }\end{array}$ \\
\hline 6 & $68 /$ female (1) & $1 / 16 / 80$ & Pennsylvania, USA & Blood & $\begin{array}{l}\text { Agnogenic } \\
\text { myeloid } \\
\text { metaplasia }\end{array}$ & Fever & Cephalexin, tobramycin \\
\hline 7 & $86 /$ female (1) & $7 / 15 / 80$ & Connecticut, USA & Blood & Near-drowning & Pneumonia & Oxacillin, gentamicin \\
\hline 8 & 75/male (1) & $8 / 19 / 80$ & Connecticut, USA & Blood & Near-drowning & Pneumonia & Cephalothin, gentamicin \\
\hline 9 & 5/male (1) & $1 / 30 / 81$ & New York, USA & Blood & CGD & Fever & NA \\
\hline 10 & 12/female (1) & $9 / 13 / 81$ & California, USA & Lung biopsy & CGD & Pneumonia & Erythromycin, rifampin \\
\hline 11 & $34 /$ female (1) & $8 / 15 / 84$ & New Mexico, USA & Ascitic fluid & None & Peritonitis & Clindamycin, gentamicin \\
\hline 12 & 28/male (1) & $5 / 14 / 85$ & Virginia, USA & Blood & Near-drowning & Sepsis & NA \\
\hline 13 & 47/female (1) & $9 / 16 / 86$ & New York, USA & $\begin{array}{l}\text { Blood, } \\
\text { pericardial fluid }\end{array}$ & $\begin{array}{l}\text { Hodgkin's } \\
\text { disease }\end{array}$ & Fever, sepsis & $\begin{array}{l}\text { Erythromycin, tobramycin, trimethoprim- } \\
\text { sulfamethoxazole }\end{array}$ \\
\hline 14 & 16/male (1) & $8 / 23 / 86$ & $\begin{array}{l}\text { Massachusetts, } \\
\text { USA }\end{array}$ & $\begin{array}{l}\text { Cerebrospinal } \\
\text { fluid }\end{array}$ & CGD & Meningitis & Vancomycin, gentamicin, others \\
\hline 15 & 19/male (3) & 'Summer' & Maryland, USA & Blood & CGD & Fever & Cefotaxime, gentamicin, ciprofloxacin \\
\hline 16 & $10 /$ male $^{*}$ & $8 / 20 / 03$ & Nova Scotia & Lymph node & CGD & $\begin{array}{l}\text { Adenitis, } \\
\text { pneumonia }\end{array}$ & Ciprofloxacin \\
\hline 17 & 24/male (5) & 'Summer' & Turkey & Blood & CGD & Pneumonia, sepsis & Cefuroxime, ciprofloxacin, meropenem, others \\
\hline
\end{tabular}

*This patient is from the present report. CGD Chronic granulomatous disease; NA Not available; USA United States of America

minimum inhibitory concentration (MIC) methods performed at the IWK Health Centre (Nova Scotia) suggested TMP. SMX resistance (no zone of inhibition surrounding the TMPSMX disc and an MIC greater than $2 / 38 \mu \mathrm{g} / \mathrm{mL}$ ). MIC testing further suggested ampicillin resistance (MIC greater than $16 \mu \mathrm{g} / \mathrm{mL}$ ) and susceptibility to both cefotaxime (MIC less than $8 \mu \mathrm{g} / \mathrm{mL}$ ) and ciprofloxacin (MIC less than $0.5 \mu \mathrm{g} / \mathrm{mL}$ ).

The patient improved dramatically after starting ciprofloxacin and was discharged five days later to complete a four to six week course. At follow-up, symptoms had resolved completely, and a repeat computed tomography scan documented resolution of the initial findings.

\section{MATERIALS AND METHODS}

All English-language literature was reviewed to identify reported cases of $F$ philomiragia infection. The data extracted from each case are summarized in Table 1.

\section{RESULTS}

Seventeen cases of $F$ philomiragia infection were identified (1-5). As noted by Wenger et al (1), patients fell into three main categories: CGD patients (mean age 14 years); patients that experienced near-drownings in saltwater; and adults with hematological malignancy. Only two cases had no discernable risk factor. CGD, the main predisposing factor, was present in eight of 17 (47\%) cases. Saltwater immersions and hematological malignancies were present in five of 17 (29\%) and two of 17 (11\%) cases, respectively. Fifteen of 17 (88\%) reported cases occurred within 50 miles of a saltwater coastline, often involving direct contact with saltwater. Most cases occurred during summer or fall, both of which are peak times for recreational saltwater exposure. Only three of 17 cases occurred during the other half of the year (from November to April). Recurring clinical presentations included pneumonia (seven of 17 cases), nonlocalized fever (four of 17 cases) and sepsis (three of 17 cases). All three cases of pneumonia in CGD patients required an invasive procedure (biopsy) to recover the organism. Including the present case, three patients with CGD developed F philomiragia infection during TMP-SMX prophylaxis. While there are little published data on the susceptibility of this organism to TMP-SMX, the authors' isolate appeared to be completely resistant, exhibiting no zone of inhibition on Kirby-Bauer disc susceptibility testing.

\section{DISCUSSION}

In 1969, Yersinia philomiragia was first reported as a novel muskrat pathogen (6). Today, the organism is categorized as a Francisella species and is recognized as an opportunistic pathogen in humans. It shares unique fatty acids, marked DNA homology and phenotypic features with $F$ tularensis; both appear in the laboratory as tiny, nonmotile, strictly aerobic Gram-negative coccobacilli $(1,7)$. Both take up Gram's counterstain poorly, have weakly positive catalase tests and grow on chocolate or cysteinesupplemented agar, yielding smooth, raised, moist colonies. F philomiragia is cysteine-independent and yields white colonies (Figure 3). In contrast, F tularensis is fastidious, with colonies typically appearing gray. Identification of $F$ philomiragia may be achieved by demonstrating a positive Kovac's oxidase test (100\% of strains positive), the production of hydrogen sulfide in triple sugar iron agar (100\% positive) and delayed gelatin hydrolysis ( $80 \%$ positive). DNA amplification methods and sequencing can confirm species identification. 
Despite genetic similarities, F philomiragia infection is epidemiologically distinct from diseases due to $F$ tularensis. Neither animal nor arthropod vectors are implicated for $F$ philomiragia and although initial isolates of the organism came from freshwater, human cases have often been associated with saltwater exposure. Tularemia, in contrast, is acquired primarily through contact with infected animals or ticks or through ingestion of contaminated freshwater or meat. $F$ philomiragia is an opportunist, while most $F$ tularensis infections occur in previously healthy people.

Similar disease manifestations for the Francisella species imply shared routes of pathogenesis. While F tularensis is clearly more virulent, both organisms are facultative intracellular organisms capable of infecting reticuloendothelial tissues. Penetration of $F$ tularensis through intact or abraded skin is followed by localized inflammation. Failure to contain the infection results in spread to regional lymph nodes. Lymphohematogenous dissemination may then occur, causing the varied presentations of tularemia. The crab-induced abrasion and subsequent local adenitis observed in the present patient suggests that, like tularemia, cutaneous inoculation is also a probable route of $F$ philomiragia acquisition. This first report of lymph node involvement during $F$ philomiragia infection is directly comparable to 'glandular' tularemia. Although both species of Francisella are now recognized to cause pulmonary infections, micronodular disease as seen in the present patient has not been previously reported with either organism. Cavitating pulmonary lesions have been reported as a rare complication of tularemia - perhaps the end result of a process that started as micronodules (8). F philomiragia cases presenting with pneumonia or sepsis parallel 'pneumonic' and 'typhoidal' forms of tularemia. Meningitis and peritonitis, present in one case each of $F$ philomiragia infection, have also been reported

\section{REFERENCES}

1. Wenger JD, Hollis DG, Weaver RE, et al. Infection caused by Francisella philomiragia (formerly Yersinia philomiragia). A newly recognized human pathogen. Ann Intern Med 1989;110:888-92

2. Seger RA, Hollis DG, Weaver RE, Hitzig WH. Chronic granulomatous disease: Fatal septicemia caused by an unnamed gram-negative bacterium. J Clin Microbiol 1982;16:821-5.

3. Sicherer SH, Asturias EJ, Winkelstein JA, Dick JD, Willoughby RE. Francisella philomiragia sepsis in chronic granulomatous disease. Pediatr Infect Dis J 1997;16:420-2.

4. Polack FP, Harrington SM, Winkelstein JA, Merz WG, Willoughby RE. Recurrent Francisella philomiragia sepsis in chronic granulomatous disease. Pediatr Infect Dis J 1998;17:442-3.

5. Friis-Moller A, Lemming LE, Valerius NH, Bruun B. Problems in identification of Francisella philomiragia associated with fatal with tularemia. Because lung (or in the present case, node) biopsy has been required to isolate the organism from CGD patients with pneumonia, $F$ philomiragia infection may be more common than reported cases suggest.

The contribution of lipopolysaccharide (LPS) to the virulence of Francisella species has been examined in recent animal models (9). When characterized by LPS chemotypes, wild strains of $F$ tularensis cause rapid death following intraperitoneal injection in mice. A single $F$ tularensis bacillus has been shown to cause death in mice, guinea pigs and hamsters. In contrast, F philomiragia, which produces lipooligosaccharide, behaves like LPS-deficient F tularensis strains and has caused significantly less morbidity and mortality in animal models.

Although susceptibility testing of Francisella species remains nonstandardized, the Clinical Laboratory and Standards Institute (Pennsylvania, USA; formerly the National Committee for Clinical Laboratory Standards, or NCCLS) have formed a Working Group on Susceptibility Testing of Fastidious Organisms that may soon provide standardized breakpoints for interpretation. Until then, laboratory data should be used with caution. Therapeutic failures involving $F$ philomiragia infection have been documented, for example, despite apparent in vitro susceptibility to cephalosporins (5). While all 14 of the isolates initially described by Wenger et al (1) were susceptible to thirdgeneration cephalosporins, all produced beta-lactamase and were ampicillin-resistant. A more recent study (3) provided the first clinical report of in vitro cefotaxime resistance. To date, there have been no clinical isolates reported with resistance to ciprofloxacin, and it may be prudent to consider this agent as part of a therapeutic regimen in moderately to severely ill patients. The best therapeutic plan, however, remains contingent on early diagnosis through clinical suspicion and rapid laboratory diagnosis of this rare pathogen.

bacteremia in a patient with chronic granulomatous disease. J Clin Microbiol 2004;42:1840-2.

6. Jensen WI, Owen CR, Jellison WL. Yersinia philomiragia sp. n., a new member of the Pasteurella group of bacteria, naturally pathogenic for the muskrat (Ondatra zibethica). J Bacteriol 1969;100:1237-41.

7. Hollis DG, Weaver RE, Steigerwalt AG, Wenger JD, Moss CW, Brenner DJ. Francisella philomiragia comb. nov. (formerly Yersinia philomiragia) and Francisella tularensis biogroup novicida (formerly Francisella novicida) associated with human disease. J Clin Microbiol 1989;27:1601-8.

8. Kozak AJ, Hall WH, Gerding DN. Cavitary pneumonia associated with tularemia. Chest 1978;73:426-7.

9. Onoprienko NN, Pavlovich NV. [The role of lipopolysaccharide in toxicity of Francisella genus bacteria]. Mol Gen Mikrobiol Virusol 2003;(3):25-8. 


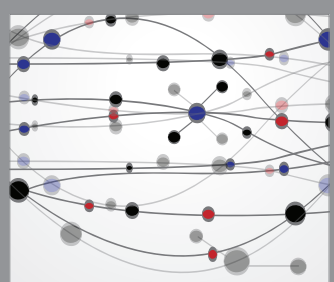

The Scientific World Journal
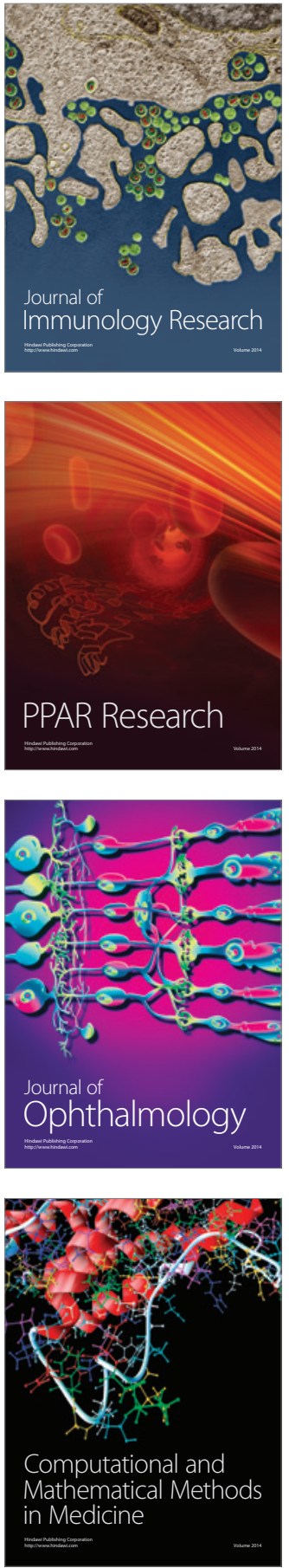

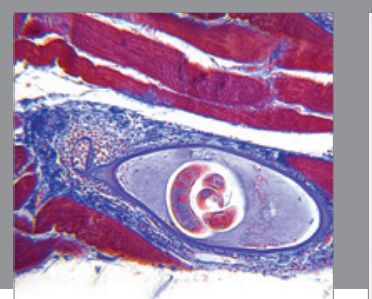

Gastroenterology Research and Practice

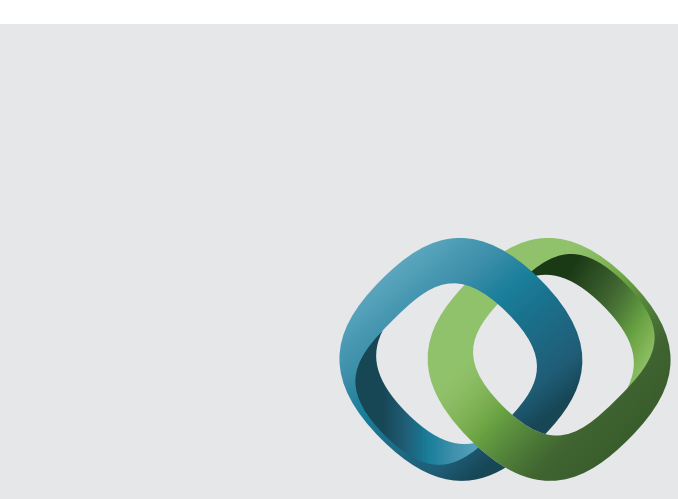

\section{Hindawi}

Submit your manuscripts at

http://www.hindawi.com
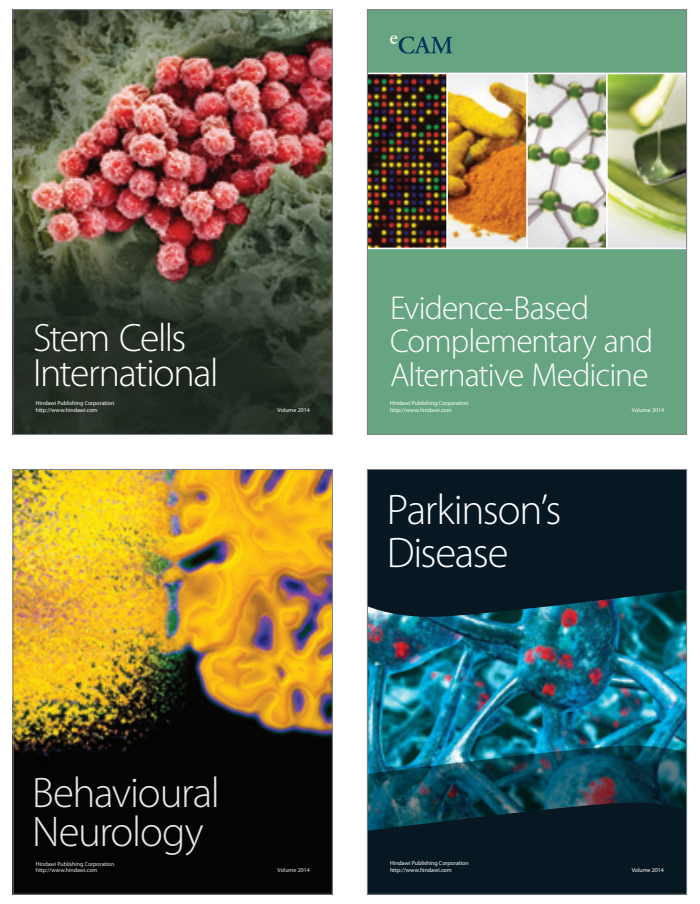
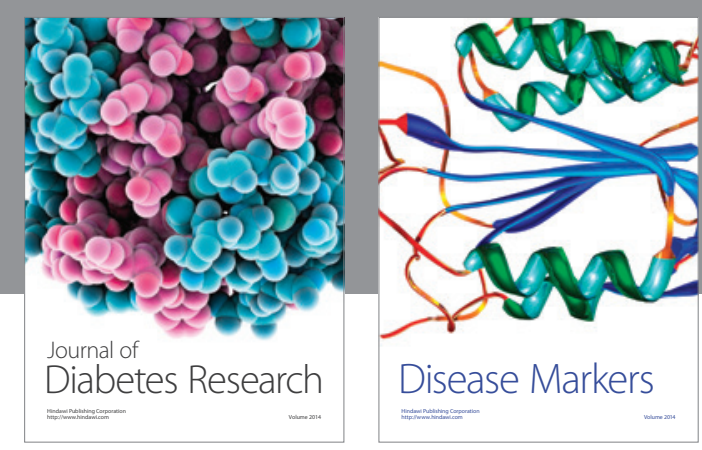

Disease Markers
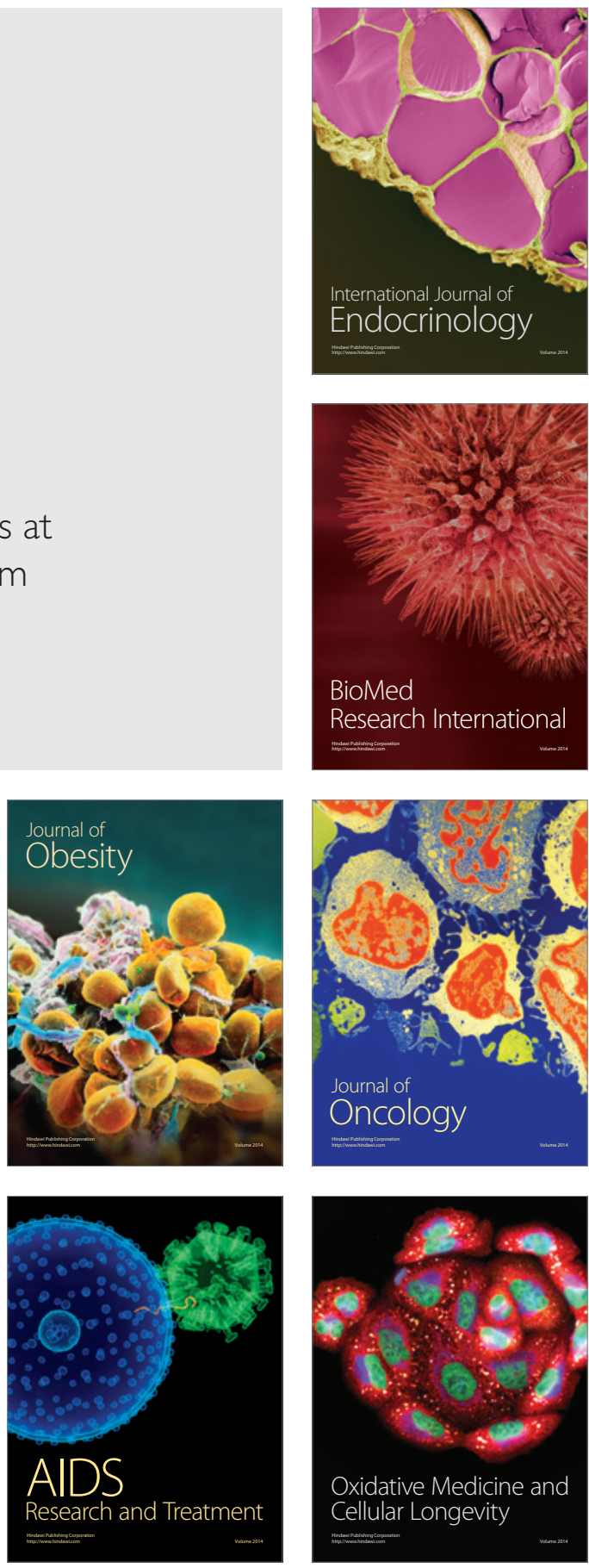